\title{
5
}

\section{Panel - The Impact of Action Research on Information Systems}

\author{
R. Baskerville \\ Binghamton University, USA
}

M. Myers

University of Auckland, New Zealand

P. A. Nielsen

Aalborg University, Denmark

\section{T. Wood-Harper \\ Salford University, $U K$}

This panel will discuss the impact of action research methodology on the field of information systems (IS). Action research is often discussed as a paragon of qualitative methods, but how has this method made a significant difference in our understanding of the interaction between information systems and the organization?

The panel will take as a point of departure Francis Lau's paper, "A Review on the Use of Action Research in Information Systems Studies." This paper analyzes a broad spectrum of published IS action research. The panel will consider the impact of this body of research along three dimensions: the impact on IS development (ISD) methods, the impact of IS research methods, and the impact on the goals and objectives of information technology practice. Richard Baskerville will open the panel with a quick overview of the history of action research. This will be followed by three brief presentations. Trevor Wood-Harper will describe the impact of action research on ISD methods. Michael Myers will focus on the impact of action research on IS research methods. Peter Axel Nielsen will describe the effects of action research on IS practice, especially focusing on the changes in Scandinavia.

Following these presentations, the audience will participate in an open discussion of the paper and the impact of this research on the field of IS. 